Homology, Homotopy and Applications, vol.10(1), 2008, pp.41-51

\title{
THE EULER CHARACTERISTIC OF A CATEGORY AS THE SUM OF A DIVERGENT SERIES
}

\author{
CLEMENS BERGER AND TOM LEINSTER
}

(communicated by J. Daniel Christensen)

\begin{abstract}
The Euler characteristic of a cell complex is often thought of as the alternating sum of the number of cells of each dimension. When the complex is infinite, the sum diverges. Nevertheless, it can sometimes be evaluated; in particular, this is possible when the complex is the nerve of a finite category. This provides an alternative definition of the Euler characteristic of a category, which is in many cases equivalent to the original one.
\end{abstract}

\section{Introduction}

What is the Euler characteristic of an infinite cell complex?

The Euler characteristic of a finite complex is most often described as the alternating sum of the number of cells of each dimension. There seems little hope of extending this formula to complexes containing infinitely many cells of the same dimension. However, there are interesting complexes in which there are only finitely many cells of each dimension, but infinitely many in total. (The classifying space of a finite group provides an example; see below.) Writing $c_{n}$ for the number of $n$-dimensional cells, we would like to find a sensible way of evaluating the divergent series $\sum_{n \in \mathbb{N}}(-1)^{n} c_{n}$, which could then be interpreted as the Euler characteristic of the complex.

To see how this might work, consider a finite group $G$. Its classifying space $B G$ is the geometric realization of a simplicial set in which an $n$-simplex is an $n$-tuple of elements of $G$. The nondegenerate $n$-simplices are the $n$-tuples of nonidentity elements, so, writing o $(G)$ for the order of $G$, there are $(\mathrm{o}(G)-1)^{n}$ of them. A simplicial set may be regarded as a kind of complex in which the cells are the nondegenerate simplices, so our task is to evaluate

$$
\sum_{n \in \mathbb{N}}(-1)^{n}(\mathrm{o}(G)-1)^{n}
$$

In the Eulerian spirit of formal calculation, we apply the formula for the sum of a geometric series, which gives the answer $1 / \mathrm{o}(G)$. And indeed, it has been established

Leinster is supported by an EPSRC Advanced Research Fellowship.

Received July 6, 2007, revised November 23, 2007; published on December 11, 2007.

2000 Mathematics Subject Classification: 18F99, 57N65, 40A05, 05C50.

Key words and phrases: Euler characteristic, finite category, divergent series, divergent sum, Möbius inversion.

This article is available at http://intlpress.com/HHA/v10/n1/a3

Copyright (c) 2007, Clemens Berger and Tom Leinster. Permission to copy for private use granted. 
that this is the 'right' value for the Euler characteristic of $G$ (or $B G$ ) from several points of view: see $[\mathbf{W}]$ and $[\mathbf{B D}]$, for instance.

Here is a first step towards making this rigorous. Take a cell complex $X$ (in some nonspecific sense; the exact meaning is not important for this discussion). Suppose that $X$ has only a finite number $c_{n}$ of cells of each dimension $n$, and write $f_{X}(t)=$ $\sum_{n \in \mathbb{N}} c_{n} t^{n}$ for the resulting formal power series. It may be that $f_{X}$ converges in some neighbourhood of 0 . If so, it may also be that $f_{X}$ can be analytically continued to -1 , and it may even be that all such analytic continuations take the same value at -1 . We could then define the Euler characteristic of $X$ to be that value. Of course, if $X$ has only finitely many cells then the situation is very simple: $f_{X}$ is a polynomial, there is a unique analytic continuation to -1 , and its value there (namely, $f_{X}(-1)$ ) is the Euler characteristic of $X$ in the usual sense.

The purpose of this paper is to use this approach to derive a notion of the Euler characteristic of a finite category. This is achieved with the aid of the nerve construction (see $\S 2$ ), which turns a category into a simplicial set.

We will see that when $X$ is the nerve of a finite category, the power series $f_{X}$ is in fact the germ at 0 of a rational function. The question of analytic continuation is then straightforward. We can therefore carry out the plan above, and this gives us a definition of the Euler characteristic of a finite category (valid when the rational function does not have a pole at -1 ). This is called the 'series Euler characteristic' of the category $(\S 2)$.

The new notion of series Euler characteristic is to be compared with the original notion of the Euler characteristic of a finite category, introduced in $[\mathbf{L}]$. We will see that in a large and important class of finite categories the two notions agree ( $\S 3)$. However, outside this class the relationship breaks down: there are examples of finite categories for which the Euler characteristic is defined in one sense but not the other (both ways round), and further examples where both are defined but their values differ $(\S 4)$.

\section{Background: the Euler characteristic of a category}

Here are the necessary definitions from $[\mathbf{L}]$, with notation changed slightly to allow a matrix-based approach.

Given a natural number $m$ and a ring $K$, write $\operatorname{Mat}_{m}(K)$ for the ring of $m \times m$ matrices over $K$. Given a finite category $\mathbf{A}$ with objects $a_{1}, \ldots, a_{m}$, write $Z_{\mathbf{A}} \in$ $\operatorname{Mat}_{m}(\mathbb{Q})$ for the matrix whose $(i, j)$-entry is the number of arrows from $a_{i}$ to $a_{j}$. (Of course, $Z_{\mathbf{A}}$ also depends on the order in which the objects are listed.) A weighting on $\mathbf{A}$ is an $m$-tuple $w^{\bullet}=\left(w^{1}, \ldots, w^{m}\right) \in \mathbb{Q}^{m}$ such that

$$
Z_{\mathbf{A}}\left(\begin{array}{c}
w^{1} \\
\vdots \\
w^{m}
\end{array}\right)=\left(\begin{array}{c}
1 \\
\vdots \\
1
\end{array}\right) .
$$

A coweighting on $\mathbf{A}$ is an $m$-tuple $w_{\bullet}=\left(w_{1}, \ldots, w_{m}\right) \in \mathbb{Q}^{m}$ such that

$$
\left(\begin{array}{lll}
w_{1} & \cdots & w_{m}
\end{array}\right) Z_{\mathbf{A}}=\left(\begin{array}{lll}
1 & \cdots & 1
\end{array}\right) .
$$

It is easy to show that if $w^{\bullet}$ is a weighting and $w_{\bullet}$ a coweighting on $\mathbf{A}$ then $\sum_{i} w^{i}=\sum_{i} w_{i}$. A finite category $\mathbf{A}$ has Euler characteristic if it admits both 
a weighting and a coweighting, and in that case its Euler characteristic $\chi(\mathbf{A})$ is $\sum_{i} w^{i}=\sum_{i} w_{i} \in \mathbb{Q}$, for any weighting $w^{\bullet}$ and coweighting $w_{\bullet}$.

The Euler characteristic of a category is independent of the choice of ordering of the objects. It is also independent of the composition and identities; that is, it depends only on the underlying directed graph. (But it is usually not equal to the Euler characteristic of the underlying graph, 'vertices minus edges'. The Euler characteristics of categories and graphs are compatible in a different sense: Proposition 2.10 of [L].)

An important special case is when $Z_{\mathbf{A}}$ is invertible. Then $\mathbf{A}$ is said to have Möbius inversion, there are a unique weighting and a unique coweighting, and $\chi(\mathbf{A})$ is the sum of the entries of $Z_{\mathbf{A}}^{-1}$.

The Euler characteristic of categories enjoys many good properties. It is invariant under equivalence and behaves predictably with respect to products, fibrations, etc. It is also compatible with Euler characteristics of other types of structure, including topological spaces, graphs, posets, groups, manifolds, and orbifolds.

\section{Related work}

The observation that the Euler characteristic of a finite group can be calculated by formal summation of the geometric series (1) is probably nearly as old as the concept of the classifying space of a group. (We learned it from a talk of John Baez.) A group can be regarded as a one-object category in which all morphisms are invertible; from that viewpoint, our purpose is to extend this formal method from finite groups to finite categories.

There are other contexts in which Euler characteristic has been described using the sum of a divergent series. Several implementations of this idea are discussed by Propp $[\mathbf{P 1}$, P2]. For finitely generated groups, the Euler characteristic is related to the growth function: see $[\mathbf{F P}]$ and $[\mathbf{G}]$, for instance. In $[\mathbf{B e}]$, the Euler characteristic of an Eilenberg-Mac Lane space of type $K(\pi, n)$ for any finite abelian group $\pi$ and any $n \geqslant 1$ is identified with the sum of a divergent series, using a particular cellular model of this space. There are also homological methods that provide a sensible way of assigning an Euler characteristic to certain infinite groups, as discussed in Chapter IX of $[\mathbf{B r}]$.

A more restrictive notion of Möbius inversion for categories is considered in [CLL] and $[\mathbf{H}]$. Like our notion, it generalizes the poset Möbius inversion of Hall, Rota, Ward and Weisner. See $\S 4$ of $[\mathbf{L}]$ for a description of the relationships between these theories. Hall's theorem - which states that for elements $a<b$ of a finite poset $P$, the value $\mu(a, b)$ of the Möbius function is the reduced Euler characteristic of the open interval $] a, b$ [ in $P$-follows easily from the proof of Theorem 2.2 by evaluating the formal power series $f_{] a, b[}$ at -1 . Compare $[\mathbf{S}, 3.8 .5]$ and $[\mathbf{L}, 1.5,4.5]$.

For a discussion of other related work, and further references, see the introduction to $[\mathbf{L}]$.

\section{The series Euler characteristic of a category}

In this section we define the series Euler characteristic of a finite category, see how the definition works in the motivating case where the category is a group, and find a way to calculate it. 
First let us recall some facts about formal power series. For any field $K$, there is a commutative diagram

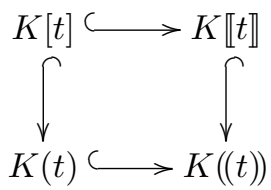

of rings. Here $K[t]$ is the ring of polynomials over $K$ and $K \llbracket t \rrbracket$ is the ring of formal power series $\sum_{n \in \mathbb{N}} a_{n} t^{n}\left(a_{n} \in K\right)$. These are both integral domains, and their fields of fractions are in the bottom row: the field $K(t)$ of rational expressions over $K$, and the field $K((t))$ of formal Laurent series over $K$ (expressions $\sum_{n \in \mathbb{Z}} a_{n} t^{n}$ such that $\left\{n \leqslant 0 \mid a_{n} \neq 0\right\}$ is finite).

The canonical inclusions of $K \llbracket t \rrbracket$ and $K(t)$ into $K((t))$ make it possible to ask whether a formal power series 'is rational'; in other words, whether the element of $K((t))$ corresponding to the power series is in the image of $K(t)$. When $K$ is a subfield of $\mathbb{C}$, the following analytic criterion applies. Let $f \in K \llbracket t \rrbracket$ and let $p, q \in K[t]$ be coprime polynomials. Then $f=p / q$ in $K((t))$ if and only if there is a neighbourhood $U$ of 0 in $\mathbb{C}$ such that for all $z \in U, q(z) \neq 0$ and $f(z)$ converges to $p(z) / q(z)$.

We will also need some notation for matrices. Let $m \in \mathbb{N}$ and let $K$ be a commutative ring. We write $s$ : $\operatorname{Mat}_{m}(K) \rightarrow K$ for the $K$-linear map defined by $s(M)=$ $\sum_{\text {by }} M_{i j}$. Every matrix $M \in \operatorname{Mat}_{m}(K)$ has an adjugate $\operatorname{adj}(M) \in \operatorname{Mat}_{m}(K)$, defined $(\operatorname{adj}(M))_{i j}=(-1)^{i+j} \cdot \operatorname{det}(M$ with its $j$ th row and $i$ th column deleted $)$

and satisfying

$$
M \cdot \operatorname{adj}(M)=\operatorname{adj}(M) \cdot M=\operatorname{det}(M) \cdot I .
$$

Lemma 2.1. Let $M$ be a square matrix over a field $K$. Then $\sum_{n \in \mathbb{N}} s\left(M^{n}\right) t^{n} \in K \llbracket t \rrbracket$ is rational.

Proof. Write

$$
F(t)=\sum_{n \in \mathbb{N}} M^{n} t^{n} \in \operatorname{Mat}_{m}(K \llbracket t \rrbracket)
$$

Then

$$
(I-M t) F(t)=I
$$

so

$$
\operatorname{det}(I-M t) \cdot F(t)=\operatorname{adj}(I-M t) .
$$

Applying the $K \llbracket t \rrbracket$-linear map $s: \operatorname{Mat}_{m}(K \llbracket t \rrbracket) \rightarrow K \llbracket t \rrbracket$,

$$
\operatorname{det}(I-M t) \cdot s(F(t))=s(\operatorname{adj}(I-M t)) .
$$

But $s(F(t))=\sum s\left(M^{n}\right) t^{n}$, and $\operatorname{det}(I-M t)$ is not the zero polynomial (since its value at $t=0$ is 1$)$, so $\sum s\left(M^{n}\right) t^{n}$ is rational and equal to

$$
\frac{s(\operatorname{adj}(I-M t))}{\operatorname{det}(I-M t)} \in K(t) .
$$


Given a simplicial set $X$ with only finitely many simplices of each dimension, let $c_{n}$ be the number of nondegenerate $n$-simplices and $f_{X}(t)=\sum_{n \in \mathbb{N}} c_{n} t^{n} \in \mathbb{Q} \llbracket t \rrbracket$. Recall that the nerve $N \mathbf{A}$ of a category $\mathbf{A}$ is a simplicial set in which an $n$-simplex is a chain

$$
x_{0} \stackrel{\phi_{1}}{\longrightarrow} x_{1} \stackrel{\phi_{2}}{\longrightarrow} \cdots \stackrel{\phi_{n}}{\longrightarrow} x_{n}
$$

of arrows in $\mathbf{A}$; such an $n$-simplex is degenerate if and only if some $\phi_{i}$ is an identity. When $\mathbf{A}$ is finite, write $f_{\mathbf{A}}=f_{N \mathbf{A}} \in \mathbb{Q} \llbracket t \rrbracket$.

Theorem 2.2. For any finite category $\mathbf{A}$, the formal power series $f_{\mathbf{A}}$ is rational (over $\mathbb{Q}$ ).

Proof. Order the objects of $\mathbf{A}$ as $a_{1}, \ldots, a_{m}$ and let $Z_{\mathbf{A}}$ be the matrix of $\mathbf{A}$ with respect to this ordering, as in $\S 1$. For each $i$ and $j$, the number of nonidentity arrows from $a_{i}$ to $a_{j}$ is $\left(Z_{\mathbf{A}}-I\right)_{i j}$. The number of nondegenerate $n$-simplices (2) beginning at $a_{i}$ and ending at $a_{j}$ is, therefore, $\left(\left(Z_{\mathbf{A}}-I\right)^{n}\right)_{i j}$. Hence the total number $c_{n}$ of nondegenerate $n$-simplices is $s\left(\left(Z_{\mathbf{A}}-I\right)^{n}\right)$. The result now follows from the lemma.

The series $\sum(-1)^{n} c_{n}$ is in general divergent. (Proposition 2.11 of $[\mathbf{L}]$ gives exact conditions for it to converge.) Nevertheless, the theorem provides a way to 'evaluate' it, returning an answer $f_{\mathbf{A}}(-1) \in \mathbb{Q} \cup\{\infty\}$.

Definition 2.3. A finite category A has series Euler characteristic if $f_{\mathbf{A}}(-1) \in$ $\mathbb{Q}$. In that case, its series Euler characteristic is $\chi_{\Sigma}(\mathbf{A})=f_{\mathbf{A}}(-1)$.

The proofs tell us that

$$
f_{\mathbf{A}}(t)=\frac{s\left(\operatorname{adj}\left(I-\left(Z_{\mathbf{A}}-I\right) t\right)\right)}{\operatorname{det}\left(I-\left(Z_{\mathbf{A}}-I\right) t\right)} .
$$

Example 2.4. Let $G$ be a monoid of finite order o $(G)$, and let $\mathbf{A}$ be the corresponding one-object category. Then $\sum(-1)^{n} c_{n}=\sum(-1)^{n}(\mathrm{o}(G)-1)^{n}$ is divergent (unless $G$ is trivial), but the rational function

$$
f_{\mathbf{A}}(t)=\sum_{n \in \mathbb{N}} c_{n} t^{n}=\frac{1}{1-(\mathrm{o}(G)-1) t}
$$

has value $1 / \mathrm{o}(G)$ at $t=-1$. Hence $\chi_{\Sigma}(\mathbf{A})=1 / \mathrm{o}(G)$.

A change of variable will be useful. Put $u=1+1 / t$ and write

$$
g_{\mathbf{A}}(u)=\frac{s\left(\operatorname{adj}\left(Z_{\mathbf{A}}-u I\right)\right)}{\operatorname{det}\left(Z_{\mathbf{A}}-u I\right)} \in \mathbb{Q}(u) .
$$

Then $f_{\mathbf{A}}(t)=(1-u) g_{\mathbf{A}}(u)$. Hence $\chi_{\Sigma}(\mathbf{A})=g_{\mathbf{A}}(0)$, with one side defined if and only if the other is. 
We will need to be able to compute values of $\chi_{\Sigma}$. The key observation is that for an $m \times m$ matrix $M$ over a commutative ring,

$$
s(\operatorname{adj}(M))=\sum_{\sigma \in S_{m}} \operatorname{sgn}(\sigma) \cdot F\left(\left(M_{i, \sigma(i)}\right)_{i \in \mathbf{m}}\right)
$$

where $\mathbf{m}=\{1, \ldots, m\}$ and $F$ is the symmetric function defined by

$$
F\left(\left(x_{i}\right)_{i \in I}\right)=\sum_{i \in I} \prod_{j \in I \backslash\{i\}} x_{j}
$$

for any finite family $\left(x_{i}\right)_{i \in I}$. This follows from the analogous formula for determinants.

Given an $m \times m$ matrix $M$ and a subset $R$ of $\mathbf{m}$, denote by $M^{[R]}$ the matrix obtained from $M$ by deleting the $i$ th row and $i$ th column for every $i \in R$. Write $\operatorname{Sym}(S)$ for the group of permutations of a set $S$.

Proposition 2.5. Let $m \in \mathbb{N}$ and let $Z$ be an $m \times m$ matrix over a commutative ring. Then

$$
\operatorname{det}(Z-u I)=\sum_{r=0}^{m}(-1)^{r} d_{r} u^{r} \quad \text { where } \quad d_{r}=\sum_{R \subseteq \mathbf{m},|R|=r} \operatorname{det}\left(Z^{[R]}\right)
$$

and

$$
s(\operatorname{adj}(Z-u I))=\sum_{r=0}^{m}(-1)^{r} e_{r} u^{r} \quad \text { where } \quad e_{r}=\sum_{R \subseteq \mathbf{m},|R|=r} s\left(\operatorname{adj}\left(Z^{[R]}\right)\right) .
$$

Proof. Equation (3) is classical $[\mathbf{J}]$. For (4), if $\sigma \in S_{m}$ then, by a short calculation,

$$
F\left(\left((Z-u I)_{i, \sigma(i)}\right)_{i \in \mathbf{m}}\right)=\sum_{R \subseteq \operatorname{Fix}(\sigma)}(-u)^{|R|} F\left(\left(Z_{i, \sigma(i)}\right)_{i \in \mathbf{m} \backslash R}\right) .
$$

Hence

$$
\begin{aligned}
s(\operatorname{adj}(Z-u I)) & =\sum_{\sigma \in S_{m}} \operatorname{sgn}(\sigma) \cdot F\left(\left((Z-u I)_{i, \sigma(i)}\right)_{i \in \mathbf{m}}\right) \\
& =\sum_{\sigma \in S_{m}, R \subseteq \operatorname{Fix}(\sigma)} \operatorname{sgn}(\sigma) \cdot(-u)^{|R|} F\left(\left(Z_{i, \sigma(i)}\right)_{i \in \mathbf{m} \backslash R}\right) \\
& =\sum_{R \subseteq \mathbf{m}}(-u)^{|R|} \sum_{\sigma^{\prime} \in \operatorname{Sym}(\mathbf{m} \backslash R)} \operatorname{sgn}\left(\sigma^{\prime}\right) \cdot F\left(\left(Z_{i, \sigma^{\prime}(i)}\right)_{i \in \mathbf{m} \backslash R}\right) \\
& =\sum_{R \subseteq \mathbf{m}}(-u)^{|R|} s\left(\operatorname{adj}\left(Z^{[R]}\right)\right),
\end{aligned}
$$

as required. (In fact, the same argument proves (3) too, by changing $s(\operatorname{adj}(-))$ to det and $F$ to product throughout.)

Given a finite category $\mathbf{A}$, take $Z=Z_{\mathbf{A}}$ and write $d_{r}^{\mathbf{A}}=d_{r}, e_{r}^{\mathbf{A}}=e_{r}$. Denote by $l$ the least number such that $d_{l}^{\mathbf{A}} \neq 0$. Then $\mathbf{A}$ has series Euler characteristic if and only if $e_{r}^{\mathbf{A}}=0$ for all $r<l$, and in that case,

$$
\chi_{\Sigma}(\mathbf{A})=e_{l}^{\mathbf{A}} / d_{l}^{\mathbf{A}} .
$$




\section{Positive results}

Among the finite categories that have Euler characteristic, those with Möbius inversion form an important class. Any finite category equivalent to one with Möbius inversion also has Euler characteristic, and this larger class encompasses most of the examples in $[\mathbf{L}]$ : finite monoids, groupoids, posets, categories in which the only endomorphisms are automorphisms (or equivalently, the only idempotents are identities), and categories admitting an epi-mono factorization system. We show that Euler characteristic and series Euler characteristic agree on this class.

Lemma 3.1. Let $M$ be a square matrix such that for some $i \neq j$, the ith and $j$ th rows are equal and the $i$ th and $j$ th columns are equal. Then $s(\operatorname{adj}(M))=0$.

Proof. First suppose that $i=1$ and $j=2$. Then every entry of $\operatorname{adj}(M)$ is zero except perhaps for the four in the top-left corner, which are

$$
\left(\begin{array}{cc}
y & -y \\
-y & y
\end{array}\right)
$$

where $y$ is the $(1,1)$-minor of $M$. Hence $s(\operatorname{adj}(M))=0$.

The general case is handled similarly. Alternatively, it may be reduced to the case $(i, j)=(1,2)$ by showing that $s(\operatorname{adj}(M))$ is unchanged when a permutation is applied to both the rows and the columns of $M$.

Theorem 3.2. Let $\mathbf{A}$ be a finite category equivalent to some category with Möbius inversion. Then $\mathbf{A}$ has both Euler characteristic and series Euler characteristic, and $\chi(\mathbf{A})=\chi_{\Sigma}(\mathbf{A})$.

Proof. Order the objects of $\mathbf{A}$ so that the isomorphism classes are grouped together:

$$
a_{1}^{1}, \ldots, a_{1}^{q_{1}}, \ldots, a_{n}^{1}, \ldots, a_{n}^{q_{n}}
$$

where $a_{i}^{j} \cong a_{i^{\prime}}^{j^{\prime}}$ if and only if $i=i^{\prime}$, and where each $q_{i}$ is nonzero. Let $\mathbf{B}$ be the full subcategory $\left\{a_{1}^{1}, a_{2}^{1}, \ldots, a_{n}^{1}\right\}$, a skeleton of $\mathbf{A}$. Now $\mathbf{A}$ is equivalent to some category $\mathbf{B}^{\prime}$ with Möbius inversion, and any category with Möbius inversion is skeletal, so $\mathbf{B}^{\prime}$ is isomorphic to $\mathbf{B}$ and $\mathbf{B}$ has Möbius inversion. Hence $\chi(\mathbf{B})$ is defined. Since Euler characteristic is invariant under equivalence, $\chi(\mathbf{A})$ is also defined and $\chi(\mathbf{A})=\chi(\mathbf{B})$.

Let $R \subseteq\left\{1, \ldots, q_{1}+\cdots+q_{n}\right\}$. By Lemma $3.1, s\left(\operatorname{adj}\left(Z_{\mathbf{A}}^{[R]}\right)\right)=0$ unless $R$ omits at most one element of each isomorphism class, and in particular has at least $l=$ $q_{1}+\cdots+q_{n}-n$ elements. So $e_{r}^{\mathbf{A}}=0$ for all $r<l$. If $R$ has $l$ elements then in order for $s\left(\operatorname{adj}\left(Z_{\mathbf{A}}^{[R]}\right)\right)$ to be nonzero, $R$ must omit exactly one element of each isomorphism class, in which case $Z_{\mathbf{A}}^{[R]}=Z_{\mathbf{B}}$. Hence

$$
e_{l}^{\mathbf{A}}=q_{1} \cdots q_{n} s\left(\operatorname{adj}\left(Z_{\mathbf{B}}\right)\right) .
$$

Similarly, $d_{r}^{\mathbf{A}}=0$ for all $r<l$ and

$$
d_{l}^{\mathbf{A}}=q_{1} \cdots q_{n} \operatorname{det}\left(Z_{\mathbf{B}}\right) .
$$

But $\mathbf{B}$ has Möbius inversion, that is, $\operatorname{det}\left(Z_{\mathbf{B}}\right) \neq 0$, so $d_{l}^{\mathbf{A}} \neq 0$. Hence

$$
\chi_{\Sigma}(\mathbf{A})=e_{l}^{\mathbf{A}} / d_{l}^{\mathbf{A}}=s\left(\operatorname{adj}\left(Z_{\mathbf{B}}\right)\right) / \operatorname{det}\left(Z_{\mathbf{B}}\right)=s\left(Z_{\mathbf{B}}^{-1}\right)=\chi(\mathbf{B})=\chi(\mathbf{A}),
$$

using (5) in the first equality. 
The next result gives a class of categories that need not have Euler characteristic, but do have series Euler characteristic.

Proposition 3.3. If $\mathbf{A}$ has either a weighting or a coweighting and $Z_{\mathbf{A}}$ is diagonalizable then A has series Euler characteristic.

Proof. We may write $Z_{\mathbf{A}}=P D P^{-1}$, with $D$ the diagonal matrix on $\left(\lambda_{1}, \ldots, \lambda_{m}\right)$, and by duality we may assume that $\mathbf{A}$ has a coweighting $w_{\bullet}$.

For $n \in \mathbb{N}$,

$$
s\left(\left(Z_{\mathbf{A}}-I\right)^{n}\right)=\sum_{i, j, k \in \mathbf{m}} P_{i j}\left(\lambda_{j}-1\right)^{n}\left(P^{-1}\right)_{j k}=\sum_{j \in \mathbf{m}} p_{j} p_{j}^{\prime}\left(\lambda_{j}-1\right)^{n}
$$

where $p_{j}$ is the $j$ th column-sum of $P$ and $p_{j}^{\prime}$ the $j$ th row-sum of $P^{-1}$. In the proof of Theorem 2.2 we saw that $f_{\mathbf{A}}(t)=\sum_{n} s\left(\left(Z_{\mathbf{A}}-I\right)^{n}\right) t^{n}$; hence

$$
f_{\mathbf{A}}(t)=\sum_{j \in \mathbf{m}} \frac{p_{j} p_{j}^{\prime}}{1-\left(\lambda_{j}-1\right) t} .
$$

It suffices to prove that $p_{j} p_{j}^{\prime}=0$ for all $j$ such that $\lambda_{j}=0$. Indeed, suppose that $\lambda_{j}=0$. Then, writing $P_{j}$ for the $j$ th column of $P$, we have

$$
p_{j}=\left(\begin{array}{lll}
1 & \cdots & 1
\end{array}\right) P_{j}=w_{\bullet} Z_{\mathbf{A}} P_{j}=w_{\bullet} \lambda_{j} P_{j}=0,
$$

as required.

Example 3.4. Let $\mathbf{A}$ be the 4-object category in Example 1.11(d) of [L], which admits a coweighting (since it has an initial object) but no weighting, and so does not have Euler characteristic. Then

$$
Z_{\mathbf{A}}=\left(\begin{array}{llll}
2 & 2 & 1 & 1 \\
2 & 2 & 1 & 2 \\
1 & 1 & 1 & 1 \\
0 & 0 & 0 & 1
\end{array}\right),
$$

which is diagonalizable, so $\mathbf{A}$ has series Euler characteristic. (In fact, it can be shown using (6) that $\chi_{\Sigma}(\mathbf{A})=1$.)

\section{Negative results}

We have already seen that Euler characteristic and series Euler characteristic are defined and agree in a large and important class of finite categories, namely, those equivalent to some category with Möbius inversion. In this section we see that outside this class, the relationship breaks down.

We first see that the properties of having Euler characteristic and having series Euler characteristic are logically independent. In other words, all four possibilities occur: a category may have both Euler characteristic and series Euler characteristic (as in §3), series Euler characteristic but not Euler characteristic (Example 3.4), Euler characteristic but not series Euler characteristic (Example 4.3), or neither (Example 4.4). Furthermore, even when both are defined, they do not necessarily agree (Example 4.5). 
Given the disagreement between the two definitions, one might ask which (if either) should be regarded as superior. A major defect of series Euler characteristic is that it is not invariant under equivalence (Example 4.6). In contrast, ordinary Euler characteristic is invariant not only under equivalence but also under the existence of an adjunction (Proposition 2.4 of $[\mathbf{L}]$ ). We do not know whether series Euler characteristic enjoys the same properties with respect to products, fibrations, etc.

For the examples, we will need to know something about which matrices arise from categories. Let us say that a square matrix $Z$ of natural numbers is the matrix of a category if there exists a finite category $\mathbf{A}$ such that $Z=Z_{\mathbf{A}}$ (with respect to some ordering of the objects). Any such matrix $Z$ is certainly reflexive $\left(Z_{i i} \geqslant 1\right.$ for all $i)$ and transitive $\left(Z_{i j}, Z_{j k} \geqslant 1 \Longrightarrow Z_{i k} \geqslant 1\right)$. These necessary conditions are not sufficient; for instance, it can be shown that

$$
\left(\begin{array}{ll}
1 & 2 \\
1 & 2
\end{array}\right)
$$

is not the matrix of a category. (This has also been observed by Allouch [A].) However, we do have:

Lemma 4.1. Let $Z$ be a transitive square matrix of natural numbers whose diagonal entries are all at least 2 . Then $Z$ is the matrix of a category.

Proof. Suppose that $Z$ is an $m \times m$ matrix. We define a category structure on the directed graph with objects $1, \ldots, m$ and with $Z_{i j}$ arrows from $i$ to $j$, for each $i$ and $j$. For each $i$, choose an arrow $1_{i}: i \rightarrow i$. For each pair $(i, j)$ such that $Z_{i j} \geqslant 1$, choose an arrow $\phi_{i j}: i \rightarrow j$, with $\phi_{i i} \neq 1_{i}$ for all $i$. To define composition, take arrows $i \stackrel{\alpha}{\longrightarrow} j \stackrel{\beta}{\longrightarrow} k$. If either $\alpha$ or $\beta$ is an identity, it is clear how $\beta \circ \alpha$ must be defined; otherwise, put $\beta \circ \alpha=\phi_{i k}$.

Corollary 4.2. Let $Z$ be a square matrix of positive integers whose diagonal entries are all at least 2 . Then $Z$ is the matrix of a category.

All of the examples that follow use this corollary without mention. They can be verified using Proposition 2.5 and the remark after it.

Example 4.3. A category may have Euler characteristic but not series Euler characteristic. For example, there is a category $\mathbf{A}$ with

$$
Z_{\mathbf{A}}=\left(\begin{array}{cccc}
6 & 6 & 15 & 9 \\
6 & 6 & 6 & 6 \\
6 & 6 & 9 & 7 \\
6 & 30 & 9 & 15
\end{array}\right), \quad g_{\mathbf{A}}(u)=\frac{4(1+u)}{u(36-u)}
$$

Then $\mathbf{A}$ has a weighting $(1 / 6,0,0,0)$ and a coweighting $(0,1 / 6,0,0)$, so $\chi(\mathbf{A})=1 / 6$. But $g_{\mathbf{A}}$ has a pole at 0 , so $\chi_{\Sigma}(\mathbf{A})$ is undefined.

Example 4.4. A finite category may have neither Euler characteristic nor series Euler characteristic. For example, there is a category $\mathbf{A}$ with

$$
Z_{\mathbf{A}}=\left(\begin{array}{ll}
2 & 4 \\
1 & 2
\end{array}\right), \quad g_{\mathbf{A}}(u)=\frac{1+2 u}{u(4-u)} .
$$

Then A does not have a weighting or a coweighting, so certainly does not have Euler 
characteristic; $\chi_{\Sigma}(\mathbf{A})$ is also undefined.

Example 4.5. Even if a category has Euler characteristic in both senses, their values may differ. For example, there is a category $\mathbf{A}$ with

$$
Z_{\mathbf{A}}=\left(\begin{array}{lll}
2 & 2 & 2 \\
2 & 2 & 2 \\
2 & 8 & 5
\end{array}\right), \quad g_{\mathbf{A}}(u)=\frac{3}{9-u} .
$$

Then $\chi(\mathbf{A})=1 / 2$ (since $(1 / 2,0,0)$ is both a weighting and a coweighting) but $\chi_{\Sigma}(\mathbf{A})=1 / 3$.

Example 4.6. Series Euler characteristic is not invariant under equivalence. For example, we may choose a category $\mathbf{A}$ satisfying

$$
Z_{\mathbf{A}}=\left(\begin{array}{ll}
3 & 3 \\
2 & 2
\end{array}\right), \quad g_{\mathbf{A}}(u)=\frac{2}{5-u}, \quad \chi_{\Sigma}(\mathbf{A})=\frac{2}{5} .
$$

Write the objects of $\mathbf{A}$ as $a_{1}, a_{2}$ and form a new category $\mathbf{B}$ by adjoining an object $a_{3}$ isomorphic to $a_{2}$. Then $\mathbf{B}$ is equivalent to $\mathbf{A}$ and

$$
Z_{\mathbf{B}}=\left(\begin{array}{lll}
3 & 3 & 3 \\
2 & 2 & 2 \\
2 & 2 & 2
\end{array}\right), \quad g_{\mathbf{B}}(u)=\frac{3}{7-u}, \quad \chi_{\Sigma}(\mathbf{B})=\frac{3}{7} .
$$

The remaining examples concern Proposition 3.3, which gives sufficient conditions for series Euler characteristic to be defined.

Example 4.7. The proposition is sharp, in the sense that neither of its hypotheses can be dropped. Example 4.4 shows that we cannot drop the first hypothesis (that A admits a weighting or a coweighting), since there $Z_{\mathbf{A}}$ is diagonalizable but $\chi_{\Sigma}(\mathbf{A})$ is undefined. To see that we cannot drop the second hypothesis (diagonalizability of $\left.Z_{\mathbf{A}}\right)$, take the following example:

$$
Z_{\mathbf{A}}=\left(\begin{array}{ccc}
2 & 3 & 5 \\
2 & 3 & 5 \\
2 & 1 & 3
\end{array}\right), \quad g_{\mathbf{A}}(u)=\frac{2+3 u}{u(8-u)} .
$$

Then $\mathbf{A}$ has a weighting $(1 / 2,0,0)$, but $\chi_{\Sigma}(\mathbf{A})$ is undefined.

Example 4.8. Even when $\mathbf{A}$ does have a weighting and $Z_{\mathbf{A}}$ is diagonalizable, $\chi_{\Sigma}(\mathbf{A})$ need not be the total weight $\sum_{i} w^{i}$ of every weighting $w^{\bullet}$. Indeed, the total weight may vary with the weighting chosen. For example, there is a category $\mathbf{A}$ with

$$
Z_{\mathbf{A}}=\left(\begin{array}{ll}
2 & 3 \\
2 & 3
\end{array}\right), \quad g_{\mathbf{A}}(u)=\frac{2}{5-u}, \quad \chi_{\Sigma}(\mathbf{A})=\frac{2}{5} .
$$

Then $Z_{\mathbf{A}}$ is diagonalizable, and $(1 / 2,0)$ and $(0,1 / 3)$ are weightings whose total weights are different.

\section{Acknowledgements}

This work began during a visit by Leinster to the University of Nice in the spring of 2007, at the invitation of Eugenia Cheng. We thank her, along with John Baez, Arnaud Beauville, Christina Cobbold and André Hirschowitz. 


\section{References}

[A] S. Allouch, Classification des catégories finies, Mémoire de Master 2, Université de Nice, 2007.

[BD] J. Baez and J. Dolan, From finite sets to Feynman diagrams, in Mathematics Unlimited-2001 and Beyond, Springer-Verlag, New York, 2001.

[Be] C. Berger, Iterated wreath product of the simplex category and iterated loop spaces, Adv. Math. 213 (2007), 230-270.

[Br] K. S. Brown, Cohomology of Groups, Springer-Verlag, New York, 1982.

[CLL] M. Content, F. Lemay, and P. Leroux, Catégories de Möbius et fonctorialités: un cadre général pour l'inversion de Möbius, J. Combin. Theory Ser. A 28 (1980), 169-190.

[FP] W. J. Floyd and S. P. Plotnick, Growth functions on Fuchsian groups and the Euler characteristic, Invent. Math. 88 (1987), 1-29.

[G] R. I. Grigorchuk, Growth functions, rewriting systems and Euler characteristic (Russian), Mat. Zametki 58 (1995), 653-668; English translation in Math. Notes 58 (1995), 1136-1146.

[H] J. Haigh, On the Möbius algebra and the Grothendieck ring of a finite category, J. London Math. Soc. (2) 21 (1980), 81-92.

[J] N. Jacobson, Lectures in Abstract Algebra, Vol. II: Linear Algebra, D. Van Nostrand, Co., Inc., New York, 1953.

[L] T. Leinster, The Euler characteristic of a category, Documenta Mathematica, to appear, arXiv:math.CT/0610260 (2006).

[P1] J. Propp, Euler measure as generalized cardinality, arXiv:math.C0/0203289 (2002).

[P2] J. Propp, Exponentiation and Euler measure, Algebra Universalis 49 (2003), 459-471.

[S] R. P. Stanley, Enumerative Combinatorics, Vol. I, Cambridge Studies in Advanced Mathematics 49, Cambridge University Press, Cambridge, corrected reprint, 1997.

[W] C. T. C. Wall, Rational Euler characteristic, Proc. Cambridge Philos. Soc. 57 (1961), 182-184.

Clemens Berger cberger@math.unice.fr http://math1.unice.fr/ cberger

Université de Nice, Laboratoire J.-A. Dieudonné, Parc Valrose, 06108 Nice Cedex 2, France

Tom Leinster T.Leinster@maths.gla.ac.uk http://www.maths.gla.ac.uk/ tl

Department of Mathematics, University of Glasgow, University Gardens, Glasgow G12 8QW, UK 